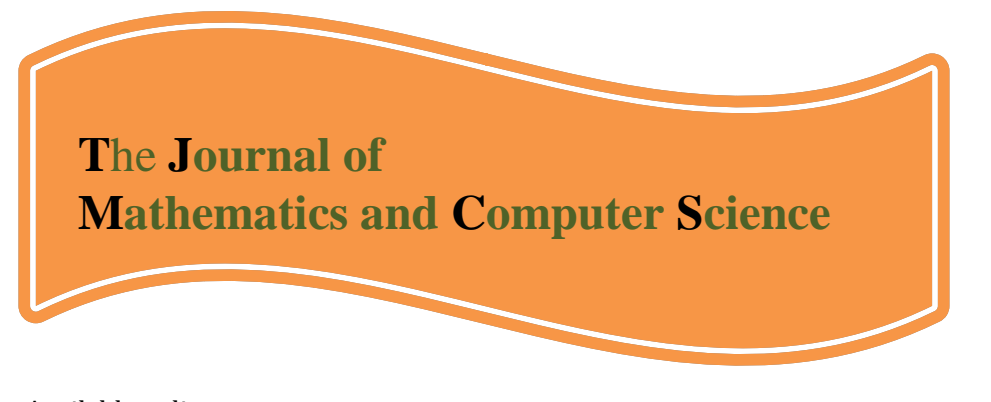

Available online at

\title{
http://www.TJMCS.com
}

The Journal of Mathematics and Computer Science Vol .5 No.1 (2012) 53-59

\section{Reduced Differential Transform Method For Solving Seventh Order Sawada-Kotera Equations}

\author{
A. Haghbin \\ Department of Mathematics, Gorgan branch, Islamic Azad University, Gorgan, Iran. \\ ahmadbin@yahoo.com \\ S. Hesam \\ Department of Mathematics, School of Mathematical Sciences, Shahrood University of \\ Technology, Shahrood, Iran \\ taranome2009@yahoo.com
}

Received: November 2012, Revised: December 2012

Online Publication: December 2012

\begin{abstract}
In this paper, reduced differential transform method (RDTM) is used to solve two different seventh-order nonlinear partial differential KdV equations. Seventh-order Sawada-Kotera (for short, sSK) and a Lax's seventh-order KdV (for short, LsKdV) equations are well known and considered for solve. reduced differential transform method can be used as an alternative to obtain analytic and approximate solutions of different types of differential equations applied in engineering mathematics. Ultimately this method is implemented to solve these equations so convenient and effective solutions can be obtained.
\end{abstract}

Keywords: Sawada-Kotera Equations; Reduced differential transform method; Initial value problem.

2010 Mathematics Subject Classification: 34A12; 83C15.

\section{Introduction.}

In the present paper we employ RDTM method for solving following equations.

$$
\begin{aligned}
& u_{t}+\left(63 u^{4}+63\left(2 u^{2} u_{x x}+u u_{x}^{2}\right)+21\left(u u_{x x x x}+u_{x x}^{2}+u_{x} u_{x x x}\right)+u_{x x x x x x}\right)_{x}=0 \\
& u_{t}+\left(35 u^{4}+70\left(2 u^{2} u_{x x}+u u_{x}^{2}\right)+7\left(2 u u_{x x x x}+3 u_{x x}^{2}+4 u_{x} u_{x x x}\right)+u_{x x x x x x}\right)_{x}=0
\end{aligned}
$$


Eq. (1) is known as the seventh order Sawada-Kotera equation [2, 4] and Eq. (2) is known as the Laxs seventh-order KdV equation [2,12] respectively. Some implementation of DTM and RDTM can be found in $[1,6,9,10,11]$. In this paper, we will apply the Reduced differential transform method for solving seventh order Sawada-Kotera equation and Laxs seventh-order KdV equation. Unlike the existing analytical methods such as the Adomian decomposition method [2], the homotopy perturbation method [3], and the variational iteration method (VIM) [3, 5], the RDTM can give exact solution for the Seventh Order Sawada-Kotera Equations and provide converged approximate solutions for the noteworthy equation without linearization, discretization, perturbation, or the calculation of the complicated Adomian polynomials. Due to the above advantages and the simple implementation of the RDTM, we obtain approximate solutions to the Seventh Order SawadaKotera Equations of high accuracy. Numerical experiments associated with two initial value problems are shown to verify the efficiency of the RDTM. The paper is organized as follows. In Section 2, theoretical aspects of the method are discussed. In Section 3, several examples with analytical solutions in two dimensional cases will be given to show the effectiveness of the proposed method. Conclusions are given in Section 4.

\section{Basic idea of RDTM}

The basic definitions of RDTM are defined as follows.

Definition 2.1. If function $u(x, t)$ is analytic and differentiated continuously with respect to time $t$ and space $x$ in the domain of interest, then let

$$
U_{k}(x)=\frac{1}{k !}\left[\frac{\partial^{k}}{\partial t^{k}} u(x, t)\right]_{t=0},
$$

where the $t$-dimensional spectrum function $U_{k}(x)$ is the transformed function. In this paper, the lowercase $u(x, t)$ represents the original function while the uppercase $U_{k}(x)$ stands for the transformed function.

Definition 2.2 The differential inverse transforms of $U_{k}(x)$ is defined as follows

$$
u(x, t)=\sum_{k=0}^{\infty} U_{k}(x) t^{k},
$$

Then combining equation (3) and (4) we can write

$$
u(x, t)=\sum_{k=0}^{\infty} \frac{1}{k !}\left[\frac{\partial^{k}}{\partial t^{k}} u(x, t)\right]_{t=0} t^{k} .
$$

From the above definitions, it is easily possible to verify that the concept of the RDTM is obtained from the power series expansion. For illustration of the proposed method, we write the sSK equation (1) in the standard operator form

$$
L(u(x, t))+R(u(x, t))+N(u(x, t))=g(x, t),
$$

with initial condition

$$
u(x, 0)=f(x)
$$

where $L=\frac{\partial}{\partial t}, R=\frac{\partial^{7}}{\partial x^{7}}$ is a linear operator which has partial derivatives, $\mathrm{N}(\mathrm{u}(\mathrm{x}, \mathrm{t}))$ is the remaining sentences, which is a nonlinear term and $\mathrm{g}(\mathrm{x}, \mathrm{t})$ is an inhomogeneous term which is equal zero. According to the RDTM and Table 1, we can construct the following iteration formula:

$$
(k+1) U_{k+1}(x)=-N^{*}\left(u_{k}\right)-R\left(U_{k}(x)\right)
$$


Table 1: Reduced differential transformation.

\begin{tabular}{|l|l|}
\hline Function Form & Transformed Form \\
\hline $\mathrm{u}(\mathrm{x}, \mathrm{t})$ & $\mathrm{U}_{\mathrm{k}}(\mathrm{x})=\frac{1}{\mathrm{k} !}\left[\frac{\partial^{\mathrm{k}}}{\partial \mathrm{t}^{\mathrm{k}}} \mathrm{u}(\mathrm{x}, \mathrm{t})\right]_{\mathrm{t}=0}$ \\
$\mathrm{w}(\mathrm{x}, \mathrm{t})=\mathrm{u}(\mathrm{x}, \mathrm{t}) \pm \mathrm{v}(\mathrm{x}, \mathrm{t})$ & $\mathrm{W}_{\mathrm{k}}(\mathrm{x})=\mathrm{U}_{\mathrm{k}}(\mathrm{x}) \pm \mathrm{V}_{\mathrm{k}}(\mathrm{x})$ \\
$\mathrm{w}(\mathrm{x}, \mathrm{t})=\alpha \mathrm{u}(\mathrm{x}, \mathrm{t})$ & $\mathrm{W}_{\mathrm{k}}(\mathrm{x})=\alpha \mathrm{U}_{\mathrm{k}}(\mathrm{x})$ \\
$\mathrm{w}(\mathrm{x}, \mathrm{y})=\mathrm{x}^{\mathrm{m}} \mathrm{t}^{\mathrm{n}}$ & $\mathrm{W}_{\mathrm{k}}(\mathrm{x})=\mathrm{x}^{\mathrm{m}} \delta(\mathrm{k}-\mathrm{n})$ \\
$\mathrm{w}(\mathrm{x}, \mathrm{y})=\mathrm{x}^{\mathrm{m}} \mathrm{t}^{\mathrm{n}} \mathrm{u}(\mathrm{x}, \mathrm{t})$ & $\mathrm{W}_{\mathrm{k}}(\mathrm{x})=\mathrm{x}^{\mathrm{m}} \mathrm{U}_{\mathrm{k}-\mathrm{r}}(\mathrm{x})$ \\
$\mathrm{w}(\mathrm{x}, \mathrm{t})=\mathrm{u}(\mathrm{x}, \mathrm{t}) \mathrm{v}(\mathrm{x}, \mathrm{t})$ & $\mathrm{W}_{\mathrm{k}}(\mathrm{x})=\sum_{\mathrm{r}=0}^{\mathrm{k}} \mathrm{V}_{\mathrm{r}}(\mathrm{x}) \mathrm{U}_{\mathrm{k}-\mathrm{r}}(\mathrm{x})=\sum_{\mathrm{r}=0}^{\mathrm{k}} \mathrm{U}_{\mathrm{r}}(\mathrm{x}) \mathrm{V}_{\mathrm{k}-\mathrm{r}}(\mathrm{x})$ \\
$\mathrm{w}(\mathrm{x}, \mathrm{t})=\mathrm{u}(\mathrm{x}, \mathrm{t}) \mathrm{v}(\mathrm{x}, \mathrm{t}) \mathrm{z}(\mathrm{x}, \mathrm{t})$ & $\mathrm{W}_{\mathrm{k}}(\mathrm{x})=\sum_{\mathrm{r}=0}^{\mathrm{k}} \sum_{\mathrm{s}=0}^{\mathrm{k}-\mathrm{r}} \mathrm{U}_{\mathrm{r}}(\mathrm{x}) \mathrm{V}_{\mathrm{s}}(\mathrm{x}) \mathrm{Z}_{\mathrm{k}-\mathrm{r}-\mathrm{s}}(\mathrm{x})$ \\
$\mathrm{w}(\mathrm{x}, \mathrm{t})=\frac{\partial^{\mathrm{r}}}{\partial \mathrm{t}^{\mathrm{r}}} \mathrm{u}(\mathrm{x}, \mathrm{t})$ & $\mathrm{W}_{\mathrm{k}}(\mathrm{x})=(\mathrm{k}+1) \ldots(\mathrm{k}+\mathrm{r}) \mathrm{U}_{\mathrm{k}+\mathrm{r}}(\mathrm{x})=\frac{(\mathrm{k}+\mathrm{r}) !}{\mathrm{k} !} \mathrm{U}_{\mathrm{k}+\mathrm{r}}(\mathrm{x})$ \\
$\mathrm{w}(\mathrm{x}, \mathrm{t})=\frac{\partial}{\partial \mathrm{x}} \mathrm{u}(\mathrm{x}, \mathrm{t})$ & $\mathrm{W}_{\mathrm{k}}(\mathrm{x})=\frac{\partial}{\partial \mathrm{x}} \mathrm{U}_{\mathrm{k}}(\mathrm{x})$ \\
\hline
\end{tabular}

Where $R\left(U_{k}(x)\right), N^{*}\left(u_{k}\right)$ are the transformations of the functions $\mathrm{R}(\mathrm{u}(\mathrm{x}, \mathrm{t})), \mathrm{N}(\mathrm{u}(\mathrm{x}, \mathrm{t}))$, respectively and $u_{k}$ is a vector of $\left\{U_{0}(x), U_{1}(x), \ldots, U_{k}(x)\right\}$.

From initial condition (7), we have

$$
U_{0}(x)=f(x),
$$

Substituting (9) into (8) and by a straight forward iterative calculations, we get the following $U_{k}(x)$ values. Then the inverse transformation of the set of values gives $\left\{U_{k}(x)\right\}_{k=0}^{n}$ approximation solution in the following form

$$
\tilde{u}_{n}(x, t)=\sum_{k=0}^{n} U_{k}(x) t^{k}
$$

where $\mathrm{n}$ is order of approximation solution. Therefore, the exact solution of problem is given by

$$
u(x, t)=\lim _{n \rightarrow \infty} \tilde{u}_{n}(x, t)
$$

\section{Illustrative Examples}

To demonstrate the effectiveness of the method we consider here equations (1) and (2) with given initial condition.

Example 3.1. Consider the sSK equation (1) with the initial condition.

$$
u(x, 0)=\frac{4 \mu^{2}}{3}\left(2-3 \tanh ^{2}(\mu x)\right),
$$

As a starting point for the solution procedure, we first take the RDTM of (1) by using Table 1, and obtain the following equation 


$$
\begin{aligned}
& (k+1) U_{k+1}(x)+252 \sum_{r=0}^{k} \sum_{s=0}^{k-r} \sum_{m=0}^{k-r-s} U_{r}(x) U_{s}(x) U_{m}(x) \frac{\partial}{\partial x} U_{k-r-s-m}(x) \\
& +63 \sum_{r=0}^{k} \sum_{s=0}^{k-r} \frac{\partial}{\partial x} U_{r}(x) \frac{\partial}{\partial x} U_{s}(x) \frac{\partial}{\partial x} U_{k-r-s}(x)+378 \sum_{r=0}^{k} \sum_{s=0}^{k-r} U_{r}(x) \frac{\partial}{\partial x} U_{s}(x) \frac{\partial^{2}}{\partial x^{2}} U_{k-r-s}(x) \\
& +126 \sum_{r=0}^{k} \sum_{s=0}^{k-r} U_{r}(x) U_{s}(x) \frac{\partial^{3}}{\partial x^{3}} U_{k-r-s}(x)+63 \sum_{r=0}^{k} \frac{\partial^{2}}{\partial x^{2}} U_{r}(x) \frac{\partial^{3}}{\partial x^{3}} U_{k-r}(x) \\
& +42 \sum_{r=0}^{k} \frac{\partial}{\partial x} U_{r}(x) \frac{\partial^{4}}{\partial x^{4}} U_{k-r}(x)+21 \sum_{r=0}^{k} U_{r}(x) \frac{\partial^{5}}{\partial x^{5}} U_{k-r}(x)+\frac{\partial^{7}}{\partial x^{7}} U_{k}(x)=0 .
\end{aligned}
$$

From the initial condition (12) we write

$$
U_{0}(x)=\frac{4 \mu^{2}}{3}\left(2-3 \tanh ^{2}(\mu x)\right),
$$

Substituting Eq. (14) into Eq. (13), we successively achieve values $U_{k}(x)$ as follows

$$
\begin{aligned}
& U_{1}(x)=-\frac{2084}{3} \mu^{9} \operatorname{sech}(\mu x)^{2} \tanh (\mu x), \\
& U_{2}(x)=\frac{262144}{9} \mu^{16}(-2+\cosh (2 \mu x)) \sec h(\mu x)^{4}, \\
& U_{3}(x)=-\frac{67108864}{81} \mu^{23} \operatorname{sech}(\mu x)^{5}(-11 \sinh (\mu x)+\sinh (3 \mu x)), \ldots
\end{aligned}
$$

Finally the differential inverse transform of $U_{k}(x)$ gives

$$
u(x, t)=\sum_{k=0}^{\infty} U_{k}(x) t^{k},
$$

We, therefore, obtain

$$
u(x, t)=\frac{4 \mu^{2}}{3}\left(2-3 \tanh ^{2}\left(\mu\left(x+\frac{256 \mu^{6}}{3} t\right)\right)\right),
$$

which is the exact solution [1] of this problem.

The graphs of the analytic and approximate solutions are depicted in Figure. 1 and 2, for $\mu=0.1$ and $x \in[-100,100]$, respectively. Table 2 shows the difference of the analytical solution and numerical solution of the absolute errors.

Table 2: The numerical results for $U_{k}(x)$ in comparison with the analytical solution $\mathrm{u}(\mathrm{x}, \mathrm{t})$, when $\mu=0.1$, for the solution of Eq. (1)

\begin{tabular}{|l|l|l|l|l|l|}
\hline$t_{i} / x_{i}$ & 0.1 & 0.2 & 0.3 & 0.4 & 0.5 \\
\hline 0.1 & 0 & 0 & 0 & $3.46945 \times 10^{-18}$ & 0 \\
\hline 0.2 & 0 & 0 & $3.46945 \times 10^{-18}$ & $3.46945 \times 10^{-18}$ & $3.46945 \times 10^{-18}$ \\
\hline 0.3 & $3.46945 \times 10^{-18}$ & 0 & $3.46945 \times 10^{-18}$ & 0 & 0 \\
\hline 0.4 & 0 & $3.46945 \times 10^{-18}$ & 0 & 0 & 0 \\
\hline 0.5 & $3.46945 \times 10^{-18}$ & $3.46945 \times 10^{-18}$ & 0 & $3.46945 \times 10^{-18}$ & $3.46945 \times 10^{-18}$ \\
\hline
\end{tabular}




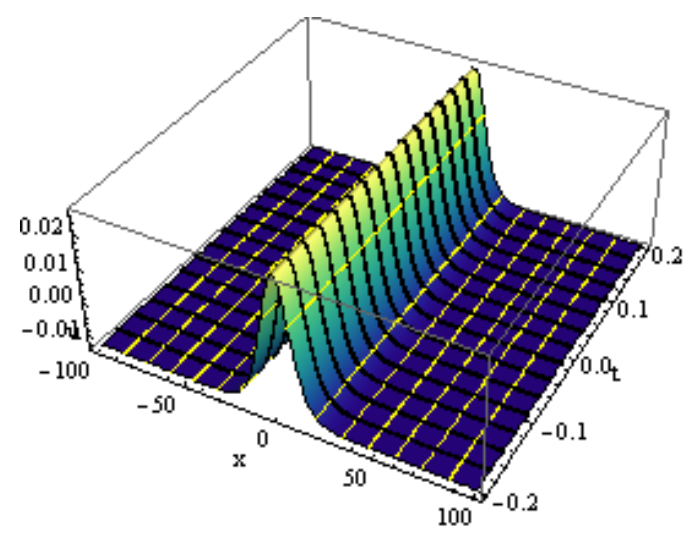

Figure 1: Exact Solution $u(x, t)$

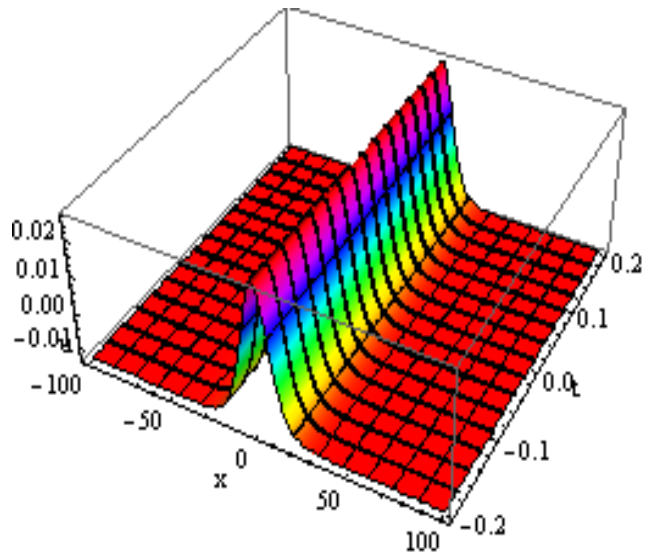

Figure 2: Approximate Solution $\mathrm{U}_{3}(\mathrm{x})$

Example 3.2. Consider the LSKdV equation with given initial condition,

$$
\left.u(x, 0)=2 \mu^{2} \operatorname{sech}^{2}(\mu x)\right),
$$

As a starting point for the solution procedure, we first take the RDTM of (1) by using Table 1, and obtain the following equation

$$
\begin{aligned}
& (k+1) U_{k+1}(x)+140 \sum_{r=0}^{k} \sum_{s=0}^{k-r} \sum_{m=0}^{k-r-s} U_{r}(x) U_{s}(x) U_{m}(x) \frac{\partial}{\partial x} U_{k-r-s-m}(x) \\
& +70 \sum_{r=0}^{k} \sum_{s=0}^{k-r} \frac{\partial}{\partial x} U_{r}(x) \frac{\partial}{\partial x} U_{s}(x) \frac{\partial}{\partial x} U_{k-r-s}(x)+280 \sum_{r=0}^{k} \sum_{s=0}^{k-r} U_{r}(x) \frac{\partial}{\partial x} U_{s}(x) \frac{\partial^{2}}{\partial x^{2}} U_{k-r-s}(x) \\
& +70 \sum_{r=0}^{k} \sum_{s=0}^{k-r} U_{r}(x) U_{s}(x) \frac{\partial^{3}}{\partial x^{3}} U_{k-r-s}(x)+70 \sum_{r=0}^{k} \frac{\partial^{2}}{\partial x^{2}} U_{r}(x) \frac{\partial^{3}}{\partial x^{3}} U_{k-r}(x) \\
& +42 \sum_{r=0}^{k} \frac{\partial}{\partial x} U_{r}(x) \frac{\partial^{4}}{\partial x^{4}} U_{k-r}(x)+14 \sum_{r=0}^{k} U_{r}(x) \frac{\partial^{5}}{\partial x^{5}} U_{k-r}(x)+\frac{\partial^{7}}{\partial x^{7}} U_{k}(x)=0 .
\end{aligned}
$$

From the initial condition (17) we write

$$
\left.U_{0}(x)=2 \mu^{2} \operatorname{sech}^{2}(\mu x)\right),
$$

Substituting Eq. (19) into Eq. (18), we successively achieve values $U_{k}(x)$ as follows

$$
\begin{aligned}
& U_{1}(x)=256 \mu^{9} \operatorname{sech}(\mu x)^{2} \tanh (\mu x), \\
& U_{2}(x)=8192 \mu^{16}(-2+\cosh (2 \mu x)) \operatorname{sech}(\mu x)^{4}, \\
& U_{3}(x)=\frac{524288}{3} \mu^{23} \operatorname{sech}(\mu x)^{5}(-11 \sinh (\mu x)+\sinh (3 \mu x)), \ldots
\end{aligned}
$$

Finally the differential inverse transform of $U_{k}(x)$ gives

$$
u(x, t)=\sum_{k=0}^{\infty} U_{k}(x) t^{k},
$$


We, therefore, obtain

$$
u(x, t)=2 \mu^{2}\left(\operatorname{sech}^{2}\left(\mu\left(x-64 \mu^{6} t\right)\right)^{2}\right) .
$$

which is the exact solution [1] of this problem.

The graphs of the analytic and approximate solutions are depicted in Figure. 3 and 4, for $\mu=0.1$ and $x \in[-100,100]$, respectively. Table 3 shows the difference of the analytical solution and numerical solution of the absolute errors.

Table 3: The numerical results for $U_{k}(x)$ in comparison with the analytical solution $\mathrm{u}(\mathrm{x}, \mathrm{t})$, when $\mu=0.1$ for the solution of Eq. (2)

\begin{tabular}{|l|l|l|l|l|l|}
\hline$t_{i} / x_{i}$ & 0.1 & 0.2 & 0.3 & 0.4 & 0.5 \\
\hline 0.1 & 0 & $3.46945 \times 10^{-18}$ & $3.46945 \times 10^{-18}$ & $3.46945 \times 10^{-18}$ & $3.46945 \times 10^{-18}$ \\
\hline 0.2 & 0 & $3.46945 \times 10^{-18}$ & $3.46945 \times 10^{-18}$ & 0 & 0 \\
\hline 0.3 & $3.46945 \times 10^{-18}$ & $3.46945 \times 10^{-18}$ & 0 & $3.46945 \times 10^{-18}$ & $3.46945 \times 10^{-18}$ \\
\hline 0.4 & $3.46945 \times 10^{-18}$ & 0 & 0 & $3.46945 \times 10^{-18}$ & 0 \\
\hline 0.5 & $3.46945 \times 10^{-18}$ & 0 & 0 & $3.46945 \times 10^{-18}$ & $3.46945 \times 10^{-18}$ \\
\hline
\end{tabular}

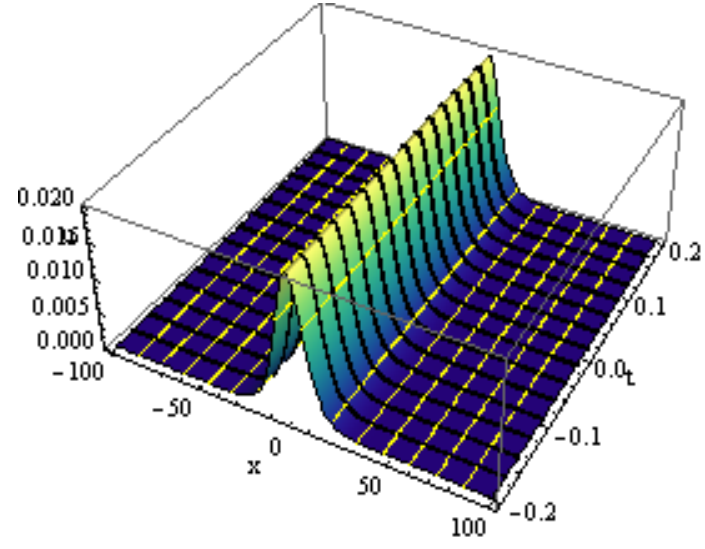

Figure 3: Exact Solution $u(x, t)$

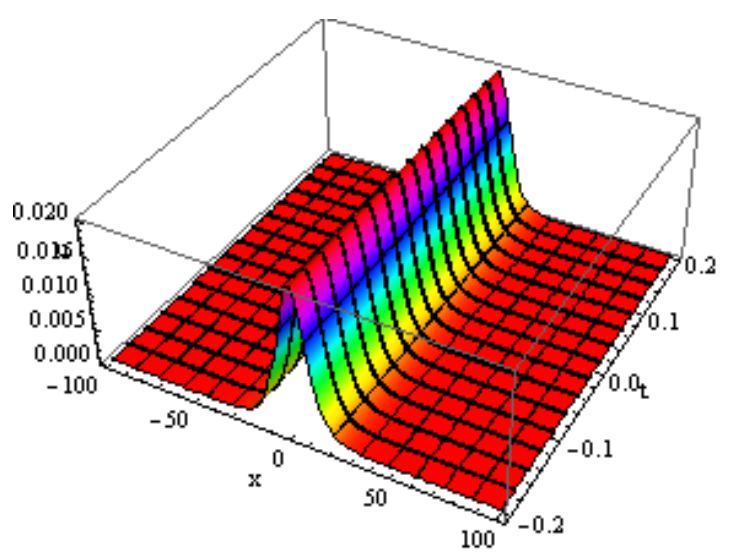

Figure 4: Approximate Solution $\mathrm{U}_{3}(\mathrm{x})$

\section{Conclusion}

In this paper, the numerical results showed that the RDTM performed well for the problems considered. The Reduced differential transform method needs less work in comparison with the traditional methods. Therefore, this method can be applied to many complicated linear and nonlinear problems and does not require linearization, discretization or perturbation.

Acknowledgement. The authors would like to express their appreciation to the research vice president of Islamic Azad University, Gorgan branch due to financial support. 


\section{References}

[1] M. Bakhshi, Mohammad Asghari-Larimi and M. Asghari-Larimi, Three- Differential transform method for solving nonlinear three-dimensional volterra integral equatons, TJMCS Vol. 4 No. 2 (2012) 246 - 256.

[2] S. M. El-Sayed and D. Kaya, An application of the ADM to seven order Sawada-Kotera equations, Appl. Math. Comput., 157 (2004) 93-101.

[3] D.D. Ganji, N. Jamshidi, Z.Z. Ganji, Hpm and Vim Methods for Finding the Exact Solutions of the Nonlinear Dispersive Equations and Seventh-Order Sawada-Kotera Equation, International Journal of Modern Physics B. Vol.23(2009) 39-52.

[4] W. Hereman, P.P. Banerjee, A. Korpel, G. Assanto, A. van Immerzeele and A. Meerpoel, Exact solitary wave solutions of nonlinear evolution and wave equations using a direct algebraic method, J. Phys. A. Math. Gen., 19 (1986) 607-628.

[5] H. Jafari, A. Yazdani, J. Vahidi, D.D. Ganji, Application of He's Variational Iteration Method for Solving Seventh Order Sawada-Kotera Equations, Appl. Math.Sci. Vol. 2(2008) No.10, 471-477.

[6] Y. Keskin, G. Oturanc, Numerical simulations of systems of PDEs by reduced differential transform method, Communications in Nonlinear Science and Numerical Simulations (In Press).

[7] Y. Keskin, G. Oturanc, Reduced Differential Transform Method for fractional partial differential equations, Nonlinear Science Letters A., 1(2) (2010), 61-72. 7900

[8] Y. Keskin, G. Oturanc, Reduced Differential Transform Method for fractional partial differential equations, Nonlinear Science Letters A .,1(2) (2010), 61-72. 7900

[9] Y. Keskin, Ph.D Thesis, Selcuk University, (2010) (in Turkish).

[10] Muhammad R. Mohyuddin, Muhammad A. Sadiq, A.M. Siddiqui, Differential Transformation Method and Variation Iteration Method for Cauchy Reaction-diffusion Problems, TJMCS Vol .1 No.2 (2010) 90-101.

[11] Mohamed I. A. Othman and A. M. S. Mahdy, Differential Transformation Method and Variation Iteration Method for Cauchy Reaction-diffusion Problems, TJMCS Vol .1 No.2 (2010) 61-75

[12] E. J. Parkes and B.R. Duffy, An automated tanh-function method for finding solitary wave solutions to non-linear evolution equations, Comput. Phys. Commun., 98 (1996) 288-300. 